\title{
Barrierefrei im Digitalen? \\ Behindertwerden sowie Möglichkeiten der Überwindung von Barrieren im Kontext des Digitalen
}

\section{Zusammenfassung}

Ausgehend von dem utopischen sowie dystopischen Potenzial, das Digitalisierungsprozessen innewohnt, werden unter dem Ziel einer nichtbehinderten Partizipation sowohl Möglichkeiten herausgearbeitet, wie im Digitalen Barrieren überwunden werden können (Transformation digitaler Medien in verschiedene Wahrnehmungs- und Kommunikationsmodi, Erleichterungen im Arbeitsalltag, transhumane Assistenzsysteme), als auch Barrieren identifiziert, die sich im Digitalen verschärft stellen (Einschränkungen durch finanzielle Möglichkeiten, diskriminierende digitale Praxen, Nichtnutzen der digitalen Möglichkeit sowie fehlende Passförmigkeit zwischen digitalen Kommunikationsmodi und den Nichtnutzer*innen). Anschließend wird diskutiert, wie diese behindernden Aspekte minimiert werden können. Der Abbau von bestehenden Barrieren braucht eine aktive, politische Gestaltung. Es ist anzuknüpfen an die Lebenskontexte der Nichtuser*innen, für die die Relevanz einer konstruktiven Nutzung der digitalen Technologien erschlossen sowie Selbstwirksamkeitserfahrungen ermöglicht werden müssen. Für die gesellschaftliche, mediale und politische Einflussnahme könnten sich die Disability Studies verstanden als Public Disability Studies verantwortlich zeichnen.

Schlüsselwörter: Digitalisierung, Behinderung, Inklusion, Barrierefreiheit, Partizipation, Heterogenität

\section{Digital Accessibility?}

Becoming Disabled and Ways of Overcoming Barriers in the Context of the Digital

\section{Abstract in English}

Based on the utopian and dystopian potential inherent in digitization processes, the paper identifies possibilities for overcoming barriers in the digital world as well as barriers that are becoming more acute in the digital world. Subsequently, it will be discussed how these disabling aspects can be minimized. The elimination of existing barriers needs an active, political approach. It must be linked to the lives of non-users, for whom the relevance of a constructive use of digital technologies must be developed and experiences of self-efficacy made possible. Disability Studies, understood as Public Disability Studies, could be responsible for influencing social, media and political sphere.

Keywords: digitization, disability, accessibility, participation, heterogeneity
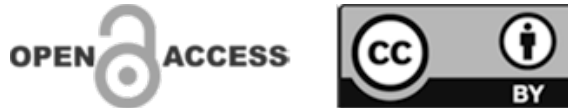


\section{Hinführung: Digitale Utopien oder Dystopien?}

Betrachtet man Digitalisierungsprozesse so lassen sich darin enthaltene utopische Elemente identifizieren, die mit der Hoffnung verbunden waren, dass digitale Technologien Gesellschaften und menschliche Lebensbedingungen verbessern könnten (Harari, 2017; Lanier, 2015; Sendler, 2018). Utopisch ist Digitalisierung im eigentlichen Wortsinn, weil sie Menschen ortsunabhängiger und auch ortloser werden lässt. Solange am konkreten Ort Strom, Internetverbindung und das erforderliche technische Equipment vorhanden sind, sind digitale Kommunikations-, Lern-, Teilhabe- oder Arbeitsprozesse möglich. Berichte von Digitalnomad*innen, die aus dem Wohnmobil heraus oder auf dem Kreuzfahrtschiff zeit- und ortsunabhängig arbeiten (Lachner, 2015; Moosmann, 2016), erscheinen als Vorzeichen einer neuen Arbeitswelt, die von Work-Life-Balance, dem Verfolgen eigener Interessen und Bedürfnisse geprägt und von einer Entfremdung oder einem Ausnutzen der Arbeitenden weit entfernt ist. Die Anfangszeit des Internets, in der eine flächendeckende Nutzung immer üblicher wurde, war geprägt von Hoffnungen auf positive gesellschaftliche Veränderungen (Thiel, 2014): Durch den freien Zugang zu Wissen oder open sourceTechnologien sollten Bildungschancen und Partizipationsmöglichkeiten für alle geschaffen werden.

Doch kritisch zurückblickend zeigt sich, dass diese Initiativen längst ihre Unschuld verloren haben. So spiegelt die Wissensplattform Wikipedia, die Inbegriff eines freien Zugangs zu Wissen ist, ebenso gesellschaftliche Machtverhältnisse wider, wenn z.B. weniger Frauen sowohl als Autorinnen als auch in Bezug auf die Inhalte repräsentiert sind (Deutschlandfunk Nova, 2018). Längst ist klar, dass Nutzer*innen für "freie Zugänge“ mit der Währung ihrer Daten zahlen (Lanier, 2014; 2015) und dass von den Privilegien der neuen Arbeitswelt einige profitieren, während andere mit katastrophalen Arbeitsbedingungen an sehr konkreten Orten, bspw. bei der Produktion technischer Geräte, die eigentliche Last dafür tragen (Karlstetter, 2018, S. 14-15). Und nicht zuletzt zeigt der zwangsweise Wechsel ins Digitale angesichts des coronabedingten Lockdowns, was bei allein digital zur Verfügung stehenden Kommunikationen verloren geht, und wie schwer es ist, im Digitalen allen eine gleichberechtigte Teilhabe zu ermöglichen, da die Zugänge und Bildungschancen, die mit der Digitalisierung einhergehen, unterschiedlich verteilt sind (DGfE, 2020). In diesen Gegenüberstellungen zeigt sich, dass Digitalisierungsprozesse sowohl ein utopisches als auch ein dystopisches Potenzial in sich tragen (Rebhorn, 2019): sie bieten einerseits die utopische Chance, menschliche Lebensbedingungen zukünftig zu verbessern. Andererseits bringen sie jedoch zahlreiche Hürden mit sich, die einer Dystopie gleichen, weil Menschen in ihrer freien Entfaltung eingeschränkt werden und sich ihre Lebensbedingungen dramatisch verschlechtern.

Ausgehend vom Themen-Schwerpunkt dieses Heftes "Utopie und Dystopie“ wird daher im vorliegenden Beitrag die Forschungsfrage erörtert, inwiefern der mit Digitalisierungsprozessen einhergehende Wandel Chancen einer behinderungsfreien Gesellschaft birgt - wodurch utopische Hoffnungen eingelöst werden können - sowie welche dystopischen Grenzen damit verbunden sind - d.h. welche behindernden Aspekte darin liegen. Damit wird die Grundfrage des Heftes, wie sich das Leben behinderter Menschen angesichts von Megatrends verändern wird, aufgegriffen und im Kontext des Digitalen diskutiert. Das folgt dem Verständnis der Disability Studies, dass Behinderung „nicht als naturgegebenes, überhistorisches Phänomen [zu sehen ist,] sondern als eine gesellschaftlich negativ bewertete Differenz, die sozial konstruiert wird und daher stets in ihrem jeweiligen historischen, sozialen und kulturellen Kontext analysiert, gedeutet und verstehbar gemacht werden muss" (Brehme et al., 2020, S. 9). Es gilt daher, Formen des Behindertwerdens im Kontext des Digitalen zu identifizieren. Dieser Frage wird in einem hermeneutisch-diskutierenden Verfahren nachgegangen, indem zuerst dargelegt wird, wie im Digitalen einerseits Barrieren überwunden werden können, und andererseits Barrieren be- und entstehen. Dabei werden die Konzepte „Barrieren“ bzw. "Behindertwerden" in einem weiten Sinne so verstanden, dass Menschen dadurch in ihrer gleichberechtigten Teilhabe eingeschränkt sind (Hirschberg, 2021).

Zudem wird ein weites Verständnis von Digitalisierung genutzt. Digitalisierung meint nicht nur, dass ein Medium, das vorher analog war, nun digital ist. Digitalisierung wird als ein gesellschaftlicher Transformationsprozess verstanden, der alle gesellschaftlichen Bereiche erfasst und "der durch den vermehrten Einsatz von digitalen Artefakten mit zunehmender Quantität, zunehmender Dynamik, Qualität und Leistungsfähigkeit, zunehmender Durchdringungstiefe in Lebenswelten und systemischen Kontexten, 
mit zunehmendem Vernetzungsgrad und zunehmender, letztlich globaler Reichweite geprägt ist" (Charbonnier, 2018, S. 239). Es wird davon ausgegangen, dass dieser Transformationsprozess letztlich alle Menschen, auch unabhängig von der eigenen Nutzung digitaler Technologien, betrifft.

\section{Ziel: Nichtbehinderte Partizipation im Digitalen sowie durch digitale Transformationen}

Um bestehende Barrieren überhaupt identifizieren und analysieren zu können, braucht es eine Verständigung darüber, woran Menschen gehindert werden, denn nur ausgehend von einer solchen „Zielvorstellung", ist es möglich, eine genauere Bestimmung vorhandener Barrieren vorzunehmen. Diese Bestimmung korreliert mit dem zu Grunde liegenden Verständnis von Behinderung und Partizipation.

Behinderung wird - sowohl der sozialen als auch der kulturellen und menschenrechtlichen Lesart folgend als kulturelles Konstrukt verstanden, bei dem hergestellte Unterschiede zu ungleichen Partizipationsmöglichkeiten und ggf. auch ungleichen Rechten führen (Gottwald, 2019). Dabei wird ein breites Verständnis von Behinderung grundgelegt. Behindert sind Menschen dann, wenn sie in ihren Partizipationsmöglichkeiten beschränkt werden. Partizipation wird im Sinne von Inklusion als „das Recht auf eine volle Zugehörigkeit" (Theunissen \& Schwalb, 2018, S. 16) in allen gesellschaftlichen Bereichen definiert.

Verknüpft man die beiden Aspekte Behinderung und Partizipation miteinander, lässt sich als grundlegendes Ziel die nichtbehinderte Partizipation formulieren (Windisch et al., 2015). D.h. dass Menschen nicht durch spezifische Voraussetzungen, Fähigkeiten oder Lebenssituationen in ihrer vollen Teilhabe behindert werden.

Auf Grund der Verbindung von digitaler und analoger Welt ist das Ziel der nichtbehinderten Partizipation im vorliegenden Beitrag in einer doppelten Weise zu betrachten: sowohl innerhalb der digitalen Medien als auch darüber hinausgehend für die durch die Digitalisierung (nicht) zur Verfügung stehenden Möglichkeiten in der analogen Welt. Denn selbst wenn Menschen digitale Medien oder Technologien nicht oder wenig nutzen, sind sie von den Auswirkungen der digitalen Transformation betroffen, wenn z.B. ein Algorithmus über ihre Kreditwürdigkeit entscheidet (Hegemann, 2019). Zudem richtet sich das Ziel der nichtbehinderten Partizipation ausgehend vom jeweiligen Individuum auf alle gesellschaftlichen Bereiche. Ein solcher breiter Fokus ist auch ausgehend vom Digitalisierungsdiskurs erforderlich, da mehr und mehr gesellschaftliche Bereiche von digitalen Transformationen durchdrungen werden.

Partizipationsbarrieren entstehen, wenn Menschen „aufgrund von stigmatisierenden Haltungen ihrer Mitmenschen und ausschließendem Interaktionsverhalten von Beteiligungsmöglichkeiten exkludiert" oder wenn sie „durch strukturell/räumliche Bedingungen von Partizipation ferngehalten “ werden (Windisch et al., 2015, S. 398). Hingegen werden Partizipationsbarrieren abgebaut, wenn Menschen „aktiv bei ihren Beteiligungsinteressen unterstützt werden“, wenn sie „mittels einer inklusiven Kultur und eines diskriminierungssensiblen Bewusstseins zu Partizipation ermutigt werden" oder wenn sie durch eine „zugängliche barrierefreie Umweltgestaltung bei einer eigenaktiven Beteiligung unterstützt werden“ (Windisch et al., 2015, S. 398).

Partizipationsgeschehen ist nicht einseitig, sondern als Wechselwirkung zu verstehen: jeder „Mensch interagiert mit seinen individuellen Fertig- und Fähigkeiten in Wechselwirkung mit den gesellschaftlichen Einstellungen und den strukturellen Umweltbedingungen. Das bedeutet, er ist von den sozialen Bedingungen abhängig, wirkt auf sie ein und hat die Möglichkeit diese zu beeinflussen" (Windisch et al. 2015, S. 396). Es bleibt daher für die Zielbestimmung der nichtbehinderten Partizipation festzuhalten, dass diese beinhaltet, dass Menschen den Auswirkungen der digitalen Transformationen nicht bloß ausgeliefert sind, sondern mit ihnen in Wechselwirkung stehen und die Transformationsprozesse mitgestalten können. 


\section{Behindertwerden im Digitalen}

Ausgangspunkt der Frage nach den Formen des Behindertwerdens ist die These von zwei gegenläufigen Tendenzen, die im Folgenden ausführlich diskutiert werden: Zum einen besteht die Chance, dass Behinderungen aus dem analogen Kontext im digitalen Kontext keine Rolle spielen. Menschen werden hier also nicht behindert, weil durch digitale Hilfsmittel analoge Barrieren abgebaut werden oder gar nicht erst vorhanden sind. Eine körperliche Beeinträchtigung muss für das Agieren in der digitalen Sphäre keine Rolle spielen, weil Kompensationsstrategien, wie z.B. eine Braillezeile, dabei helfen, dass Beeinträchtigungen nicht zu Behinderungen werden. Zudem können Menschen im Digitalen Empowermentstrategien entwickeln, politisch aktiv werden, sich vernetzen und Behinderung dekonstruieren auf Arten, die die analoge Welt nicht ermöglicht. Zum anderen zeigen sich gerade im Digitalen diskriminierende Barrieren, die Behinderung in diesem Kontext erzeugen. Ende des Jahres 2019 wurde die Praxis des Social Media-Dienstes TikTok, Menschen mit Behinderung unsichtbar zu machen, bekannt und kritisch diskutiert (Köver \& Reuter, 2019). Auch tendiert die bildliche Kommunikation in Sozialen Medien mehrheitlich zu stereotypen Normalitätsvorstellungen (Götz, 2019) und es spiegelt sich soziale Ungleichheit im Digitalen wider (Witten, 2016). Hinzu kommen die Barrieren, die darin begründet liegen, dass behinderten Menschen, gerade denjenigen, die in Einrichtungen leben, der Zugang zur entsprechenden Hardware bzw. zum Internet fehlt oder dass Programme oder Benutzungsoberflächen nicht ausreichend barrierefrei sind (Bosse et al., 2019).

Diese einleitenden Beobachtungen zeigen, dass die Transformationen, die mit der Digitalisierung einhergehen, sowohl Chancen als auch Grenzen hinsichtlich der Überwindung von Barrieren bergen. Zunächst werde ich nun herausarbeiten, worin im Digitalen die Chancen zur Überwindung von Barrieren liegen, bevor die beschränkenden Potenziale des Digitalen problematisiert werden.

\section{1 Überwindung von Barrieren}

Mit der Digitalisierung entstanden zahlreiche Möglichkeiten, Barrieren abzubauen. Sie umfassen unterschiedlichste digitale Übersetzungs-, Wahrnehmungs- und Kommunikationsmöglichkeiten, die von der Möglichkeit des Gebärdens via Videotelefonie über Software, die Bildschirminhalte in die Braillezeile transferiert oder akustische Signale in Untertitel umwandelt, oder die Möglichkeiten einer digitalen Unterstützten Kommunikation reichen. Ein digital zur Verfügung stehender Inhalt lässt sich einfacher in eine barrierefreie Form transformieren als ein analoger, sodass bessere Zugänglichkeiten entstehen. Das gilt auch für Veranstaltungsformate, die eine digitale Zuschaltung ermöglichen, und so Mobilitätsbeschränkungen verringern. Dass sich eine solche Form der Barrierefreiheit jedoch nicht, von selbst' einstellt, sondern als Ziel verfolgt werden muss, zeigt die rechtliche Verpflichtung des barrierefreien Zugangs zu Webseiten und mobilen Anwendungen öffentlicher Stellen (Europäisches Parlament, 2016).

Ähnlich werden auch in Bildungskontexten digitale Unterrichtsmedien als Chance angesehen, leichter als analoge Medien heterogenen Anforderungen zu genügen, um inklusive Lernumgebungen zu schaffen (Patzer \& Pinkwart, 2019). Die kompensatorischen Möglichkeiten helfen, Barrieren abzubauen und können eine bessere Teilhabe im Digitalen sowie durch die Möglichkeiten, sich zu informieren und zu kommunizieren auch im Analogen ermöglichen. So kommen für den wichtigen Partizipationsbereich der Arbeitswelt (Kronauer, 2015, S. 232) Metzler et al. (2020) zu dem Schluss, dass Digitalisierung „einen positiven Einfluss auf die Inklusion von Menschen mit Behinderung in der betrieblichen Personalpolitik" hat (S. 3). Denn durch die Digitalisierung sind Erleichterungen des Arbeitsalltags möglich. Dabei zeigt sich der Zusammenhang, dass Unternehmen, die bei der Digitalisierung schon weit vorangeschritten sind, "häufiger Menschen mit Behinderung als Unternehmen ohne Bezug zu Digitalisierung" (Metzler et al. 2020, S. 3) beschäftigen. Folgt man der Kontakthypothese von Gordon Allport (1971), dass der Kontakt mit Angehörigen stigmatisierter Gruppen den Abbau von Vorurteilen unterstützt, dann verbessert die Digitalisierung nicht nur die berufliche und damit auch die gesellschaftliche Teilhabe von Menschen mit Behinderung, sondern ermöglicht somit auch gesamtgesellschaftliche Veränderungen hin zu mehr Akzeptanz von Verschiedenheit und erforderlichem Barriereabbau - gleichwohl empirische Belege für diese spezifische Interpretation der Kontakthypothese noch ausstehen. 
Die Suche danach, wie Barrieren überwunden werden können, geht noch weiter. Die Möglichkeiten dazu werden unter den Stichworten Transhumanismus und Human Enhancement verhandelt und bezeichnen Diskurse um die Beseitigung , menschlicher Mängel'. Diskutiert wird die Nutzung verschiedener Technologien, um menschliche Grenzen zu überschreiten und Menschen zu optimieren (Fabricius, 2021; Strasser, 2020). Das provoziert Fragen nach dem Menschsein, u.a. dahingehend, wie weit die Optimierung gehen darf und sollte (Schenk \& Karcher, 2018). Gerade Fragen um Formen des Human Enhancement, also die Steigerung menschlicher Fähigkeiten mit Hilfe technischer und pharmakologischer Mittel (Coenen et al., 2010, S. 9), haben zu lebendigen ethischen, aber auch bildungstheoretischen Diskussionen geführt (Wimmer, 2014). Die Option, technische Hilfsmittel neuronal zu verknüpfen, schwankt zwischen schon vorhandenen und etablierten medizintechnischen Möglichkeiten, wie z.B. dem Cochlea-Implantat oder Prothesen, die über Gedanken steuerbar sein sollen, und Möglichkeiten, die zu ethisch fragwürdigen Science-FictionEntwürfen weitergedacht werden. So ermöglichen zwar z.B. neuronal gesteuerte Exo-Skelette die erforderlichen Bewegungsabläufe von bspw. querschnittsgelähmten Menschen, können aber gleichzeitig über die neuronale Schnittstelle auch in die Entscheidungsfähigkeit und -freiheit des Individuums eingreifen (Nida-Rümelin \& Weidenfeld, 2018, S. 188-197).

Unabhängig davon, wie weit transhumane Ideen weiterentwickelt werden, wo rechtlich Grenzen gesetzt werden oder wie weit manche Ideen verbreiten werden, bestehen durch die technische Unterstützung Kompensationsmöglichkeiten bzw. Verbesserungsmöglichkeiten für körperliche Gegebenheiten, die sich ohne sie beeinträchtigend auswirken können.

Zudem liegt das enthindernde Potenzial dieses Diskurses darin, dass durch die fließende Grenze zwischen Transhumanismus und assistierenden Kompensationsmöglichkeiten die Assistenzsysteme ihren potenziell stigmatisierenden Charakter verlieren. Denn die Angst vor Stigmatisierung stellt eine Barriere für die Nutzung von Assistenzsystemen dar, da Menschen lieber auf die Nutzung verzichten als durch die Nutzung als behindert oder pflegebedürftig angesehen zu werden (Bundesministerium für Gesundheit, 2013; Meyer, 2016, S. 21). Hier können technische Unterstützungssysteme, die für eine Vielzahl von Menschen attraktiv sind, weil sie ihnen Nutzen bringen und der Smartphone-Ästhetik entsprechen zur Popularität und Verbreitung assistierender Technologien beitragen. Ein förderlicher Aspekt liegt auch in der Popularität filmischer Vorbilder (Nida-Rümelin \& Weidenfeld 2018, S. 188-197). So nutzt der Marvel Comic-Held Iron Man assistierende Technologien und ermöglicht seinem Freund James Rhodes/War Machine nach einem Absturz, der zu einer Querschnittslähmung führt, mit Hilfe des in den Anzug integrierten Exo-Skeletts weiterhin als Avenger das Universum retten zu können, wie es die populären Filme The First Avenger: Civil War aus dem Jahr 2016 bzw. Avengers Endgame aus dem Jahr 2019 erzählen.

Digitalen Technologien ermöglichen also eine Vielzahl von assistierenden Kompensationsmöglichkeiten, die jedoch wiederum kritisch zu reflektieren sind. So sind sie darauf zu befragen, welche Normalisierungsvorstellungen sie transportieren, die sich zu problematischen Formen und Überbietungslogiken steigern können, und auf diese Weise ableistische Stereotype reproduzieren. Außen vor ist zudem, wer diese - nicht zuletzt auch ökonomischem Denken folgenden "Verbesserungsmöglichkeiten" nicht nutzen will oder kann. Dass dies auch ethische Fragen betrifft, zeigt sich an der Debatte um Neuro-Enhancement: Ist es z.B. "fair" oder zulässig, konzentrationsfördernde Medikamente zu nutzen und sich dadurch Vorteile zu verschaffen (Schäfer, 2015)? Bzw. in welchem Rahmen und abhängig von welchen Faktoren sind solche Eingriffe angebracht? Zudem bleibt zu bedenken, dass so behindernde Faktoren nur kompensatorisch in Bezug auf körperliche Aspekte reduziert werden, die es "auszugleichen" gälte, anstatt behindernde Umweltfaktoren in den Blick zu nehmen. Dieser Fokus auf die behindernden Barrieren erfolgt im nächsten Schritt.

\subsection{Bestehende Barrieren}

Positive Aspekte der Digitalisierung, wie die einfacher herzustellende Barrierefreiheit, werden auch ökonomisch vermarktet. So bewirbt Apple die Barrierefreiheit des iPhones (Apple, 2021), die sich jedoch lediglich auf die User Experience bezieht und massiv eingeschränkt ist durch die Barriere der Zugänglichkeit, 
die in den finanziellen Möglichkeiten der Nutzenden liegt. Es handelt sich hierbei also um eine „Barrierefreiheit", die stark davon abhängt, welche Ressourcen Nutzenden privat oder gesellschaftlich, gestützt z.B. durch die öffentliche Finanzierung, zur Verfügung stehen. Dieses Beispiel zeigt an, dass die mit den technischen Möglichkeiten einhergehenden Verbesserungen auch wiederum Beschränkungen ausgesetzt sind, was erfordert, das Zusammenspiel von analoger Lebenswelt und digitaler Partizipation zu betrachten. Denn prinzipiell vorhandene Möglichkeiten der digitalen Teilhabe bedeuten noch lange nicht, dass diese auch genutzt werden (können). Eine grundsätzliche Herausforderung zeigt sich, wenn man stärker intersektional betrachtet, wer bislang die digitalen Chancen am wenigsten nutzen kann und dadurch in seien Partizipationsmöglichkeiten eingeschränkt ist. Durch diese Analyse zeigt sich, inwiefern Menschen auf Grund bestimmter Lebenssituationen behindert werden und dass das nicht nur Menschen, die als ,beeinträchtigt' gelten, betrifft. Cirkel (2020) benennt „Ältere, gering Qualifizierte und Alleinlebende“ (S. 1) als diejenigen, die bislang digital exkludiert sind. Als besonders ausgegrenzte Gruppe, deren Situation sich in der ,CoronaKrise' noch verstärkt, weil sie nicht auf digitale Kompensationsstrategien zurückgreifen können, identifiziert er die „Digital Abseitsstehenden“, zu denen 18 Prozent der deutschen Bevölkerung gehören. Zu ihnen „gehören zum überwiegenden Teil ältere Menschen, insbesondere Seniorinnen mit geringem Bildungsgrad sind in dieser Gruppe sehr stark vertreten" (Cirkel, 2020, S. 3). Er problematisiert des Weiteren, dass eine rein quantitative Erfassung der Nutzung noch keine Aussagen über die Qualität der Nutzung zulässt, also z.B. inwiefern Datensicherheit oder eine kritische Reflexion von im Internet zu findenden Informationen möglich sind (Cirkel, 2020). Auch unter der Gruppe der Kinder und Jugendlichen ist digitale Ungleichheit zu finden. Heterogene Herkünfte spiegeln sich in heterogenen Nutzungen und Nutzungsmöglichkeiten wider (Kutscher \& Otto, 2014; Leven \& Utzmann, 2019; Wolfert \& Leven, 2019). Ein Umstand, der nicht zuletzt als massive Barriere für chancengerechte Bildung und Erziehung im coronabedingten , häuslichen Lernen' sichtbar wurde (DGfE, 2020).

Freese und Mayerle (2015) formulieren in Bezug auf digitale Teilhabe die bleibend „große Herausforderung, Menschen mit unterschiedlichem sozioökonomischem Status zu erreichen und so (politische) Beteiligung zu ermöglichen. Lebenslagen spiegeln sich im Partizipationsverhalten von Menschen deutlich wider" (Freese \& Mayerle, 2015, S. 390). Eingeschränkte Partizipationsmöglichkeiten bestehen also sowohl im Analogen als auch im Digitalen gleichermaßen, trotz Digitalisierung. Als Ursache sehen sie eine fehlende Passförmigkeit zwischen den Kommunikationsmodi, die sogenannte ,bildungsferne' Menschen oder Menschen mit Marginalisierungserfahrungen mitbringen, und den Kommunikationsformen und -wegen, wie sie im digitalen Raum etabliert sind (Freese \& Mayerle, 2015). Ausgehend von einer intersektionalen Betrachtungsweise, dass ,Behinderungszuschreibung', ,Bildungsgrad' und ,sozioökonomischer Status' sich durchaus überlagern und wechselseitig bedingen, kann daher von gravierenden Mehrfachbenachteiligungen im Digitalen ausgegangen werden.

Die bislang vorgenommene Analyse von Barrierefreiheit sowie von bestehenden Barrieren zeigt auf, dass es im Digitalen zwar vielfältige potenziell vorhandene Möglichkeiten gibt, Ent-hinderung zu unterstützen, dass diese aber durch die faktisch nutzbaren und genutzten Möglichkeiten erheblich limitiert sind - wobei Aspekte außerhalb des Digitalen, wie z.B. die zur Verfügung stehenden Ressourcen, eine erhebliche Rolle spielen. Dieses Wechselspiel zwischen Ermöglichung und Verhinderung gilt es hin zu mehr barrierefreier Teilhabe im Digitalen zu verändern. Wie das unterstützt werden kann, wird im nächsten Schritt überlegt.

\section{Reflexionen für mehr ent-hindernde Barrierefreiheit sowie digitale Teilhabe}

Die Diskussion von Chancen und Grenzen der Digitalisierung hinsichtlich vorhandener Barrierefreiheit zeigt auf, dass digitale Medien und Kommunikationsprozesse nicht per se barrierefrei sind, sondern dass eine konstruktiv-kritische Nutzung erweiterte Barrierefreiheit ermöglichen kann. Dieser These folgend sollen nun Möglichkeiten für mehr Behinderungsfreiheit und digitale Teilhabe erarbeitet werden. 


\subsection{Benachteiligende Kontexte und Faktoren identifizieren und Barrieren abbauen}

Um das oben benannte Ziel der nichtbehinderten Partizipation zu ermöglichen, sind diejenigen Kontexte und Faktoren zu identifizieren, die sich hinsichtlich dieses Ziels beschränkend auswirken. Eine solche Identifikation beginnt sehr konkret mit der Frage der Zugänglichkeit und nimmt dann immer breiter die Barrieren in den Blick, die Menschen in ihrer Partizipation benachteiligen.

Als erstes stellt sich die Frage der prinzipiellen Zugänglichkeit von digitalen Gerätschaften, Inhalten sowie Kommunikationsmöglichkeiten. Diese sind sowohl von den finanziellen Möglichkeiten abhängig als auch von einer datensicheren Nutzung, die teils nicht gegeben ist, was User*innen auch wissen, darauf aber eher resignierend reagieren (Hasebrink et al., 2019).

Doch selbst wenn diese Barriere überwunden wäre und allen Menschen eine datensichere Basisausstattung an digitaler Technik zur Verfügung stünde, würde dies noch nicht sicherstellen, dass eine gleichberechtige Partizipation im Digitalen vorhanden wäre, weil nicht gleichermaßen alle Menschen über die digitale bzw. ITliteracy (Düggeli et al., 2018) verfügen. Darin, wie der eigene Umgang mit digitalen Techniken erlebt wird, liegt ein entscheidender Schlüssel, ob die digitalen Transformationen als Chance oder als Hemmnis wahrgenommen werden (Lühr et al., 2020).

Eine der wesentlichen Barrieren liegt daher in Art und Weise der Nutzung und nicht in den zur Verfügung stehenden Möglichkeiten und schon gar nicht in einem Mangel an Förderprogrammen, die allerdings bislang zu wenig genutzt werden (Cirkel, 2020, S. 5). Um die Lücke zwischen prinzipiell bestehenden Angeboten und ihrer Nutzung zu schließen, sieht Cirkel (2020) es als erforderlich an, „angepasste Strategien zur Ausweitung des Nutzungsverhaltens zu entwickeln, die an ihren spezifischen Verhaltensweisen, Lebenskontexten und Bedürfnissen anknüpfen" (S. 5). Ob und wie die digitalen Möglichkeiten genutzt werden, liegt darin begründet, inwiefern diese als relevant und hilfreich für die eigene Lebensführung angesehen werden. Daher wirkt sich eine fehlende Passförmigkeit zwischen bestimmten Ausgangslagen und Kontexten sowie der digitalen Angebotsstruktur als Barriere aus. Digitale Partizipationsangebote oder "(politische) Bildungsangebote [müssen] lebenslagenspezifischer gestaltet sein" (Freese \& Mayerle, 2015, S. 390), um denjenigen, die sie bislang wenig nutzen, "mehr Artikulationsmöglichkeiten einzuräumen“ (Freese \& Mayerle, 2015), denn solange digitale Technologien nicht auf Bedürfnisse und Anliegen, die Menschen in bestimmten Lebenslagen haben, reagieren, werden sie auch nicht genutzt. Wenn die digitale Sphäre als sehr fern von der eigenen Lebenswelt erscheint, man nicht den Eindruck hat, Probleme, die einen unmittelbar betreffen, dort bearbeiten zu können, und dabei gesehen und gehört zu werden, werden Menschen digitale Partizipationsangebote eher nicht nutzen (Freese \& Mayerle, 2015).

Positiv formulieren ließe sich, dass Menschen digitale Partizipationsangebote oder Bildungsangebote dann nutzen werden, wenn es ihnen etwas ,bringt', das Anliegen sie unmittelbar betrifft und sie sich darin als selbstwirksam erfahren, weil sie die Erfahrung machen können, durch ihr Handeln etwas erreichen zu können (Freese \& Mayerle, 2015). Um diese Barrieren abzubauen, gilt es, Menschen zu diesen Nutzungsmöglichkeiten zu befähigen. Dafür schlagen Freese und Mayerle (2015) die "gemeinsame Bearbeitung von konflikthaften Situationen und Alltagsproblemen“, wie z.B. „Arbeitsbedingungen, Wohnungsmarktsituation, Infrastruktur im Stadtteil, u.a." (S. 390) vor und erhoffen eine Vernetzung mit anderen Menschen sowie die Erprobung neuer Handlungsformen und Aktionen. Ausgangspunkt für digitale Partizipationsprozesse ist die Verbesserung des Nahraums. Dabei müssen die Angebote möglichst zugänglich sein, „zum Beispiel durch Komplexitätsreduzierung und die Verwendung von Bildsprache sowie Leichter Sprache“ (Freese \& Mayerle, 2015, S. 391).

Diese Initiativen sind noch weiter zu stärken, um die Voraussetzungen für breite Partizipation zu schaffen.

Das heißt Barrierefreiheit darf nicht nur in einem Sinne verstanden werden, dass digitale Inhalte transformiert und dadurch besser zugänglich werden, sondern grundsätzlich ist die Frage danach, wo ein Mehrwert, wo Relevanzen für User*innen liegen, entscheidend (Domsgen, 2019). Angebote sind entsprechend so auszurichten, dass sie Relevanz- und Selbstwirksamkeitserfahrungen ermöglichen. Dazu 
gehört der basale Aspekt, dass digitale Gerätschaften benutzungsfreundlich, störungsfrei und robust sein müssen (Cirkel, 2020).

Des Weiteren ist eine intersektionale Sichtweise erforderlich. Behindernde Heterogenitätsdimensionen sind entsprechend breit zu analysieren und in der Konzeption von Angeboten weiterhin zu bedenken. Tillmann (2014) hat bspw. geschlechtshomogene digitale Räume als Ermöglichungsräume für Emanzipation identifiziert. Ohne zuschreibende Gruppenkonstitutionen vornehmen zu wollen, ist darüber nachzudenken, inwiefern digitale Räume, in denen Menschen „unter sich“ sein können, noch mehr anzubieten wären, um heterogene Bedürfnisse, Nutzungsweisen und Relevanzen aufzugreifen und passförmige Räume zu schaffen. Dabei ist auch zu bedenken, inwiefern diese Schaffung von Peer-Netzwerken und -Räumen eine Aufgabe wäre, die seitens der Öffentlichkeit und Politik stärker wahrgenommen werden müsste, weil nicht zu erwarten ist, dass gewinnorientierte Konzerne sich ihrer von allein annehmen. Des Weiteren ist als behindernder Kontext die mit der Nutzung möglicherweise einhergehende Defizitorientierung zu benennen, womit auch das Problem der Reifizierung von Heterogenität (Grümme, 2017) verbunden ist. Produkte oder Anwendungen, die an eigene - möglicherweise auch erst in der Zukunft auftretende - Defizite erinnern, werden eher weniger akzeptiert und "Technologien und Devices, die objektiv dazu dienen, Defizite auszugleichen, werden eher akzeptiert, wenn es gelingt, sie mit dem Nimbus des Komfort- oder LifestyleProduktes zur Steigerung der Lebensqualität zu versehen, ohne Funktionalität, Nutzerfreundlichkeit und Alltagstauglichkeit zu vernachlässigen“" (Cirkel, 2020, S. 5).

Zusammenfassend lässt sich zum Abbau von Benachteiligungen sagen, dass die identifizierten Barrieren durch unterschiedliche Aspekte verursacht sind und dadurch auch hinsichtlich ihres Abbaus unterschiedlich bearbeitet werden müssen.

Dabei sind zwei „Logiken“, die zu Barrieren führen können, zu bearbeiten: Zum einen spiegelt sich in der digitalen Welt wider, was an Vorstellungen von "Normalität", "Macht" oder "Schönheit" ebenso in der analogen Welt anzutreffen ist (Witten, 2016). Zum anderen gibt es spezifisch digitale Barrieren, die z.B. durch die Betreiber*innen bzw. die Logik der Plattformen entstehen. Wie können diese jeweils abgebaut werden? Dazu werden zwei Thesen formuliert. Die erste Art von Barrieren ist vor allem durch einen aufklärerischgesellschaftlichen Diskurs, der öffentlich oder im Bildungsbereich geführt wird, zu bearbeiten. Dies gilt grundsätzlich auch für die zweite Art der Barriere, die überdies aber auch durch entsprechende Gesetzgebungen und das Einhalten der schon bestehenden Gesetze zu bearbeiten wäre, wie z.B. der EURichtlinie zur Barrierefreiheit von Webseiten (Europäisches Parlament, 2016). Damit ist auch die Öffentlichkeit adressiert, die im Kontext noch zu entwickelnder Public Disability Studies in den Blick genommen werden soll.

\section{2 „Public Disability Studies“}

Die Analyse der verschiedenen Barrieren zeigte, dass wesentliche Barrieren eher "weicher" Natur sind und als Einstellungen oder Haltungen charakterisiert werden können. Um diese zu verändern, reicht es nicht, die gesetzlichen Rahmenbedingungen zu novellieren, sondern die damit verbundene Aufgabe stellt im Wesentlichen eine Kommunikationsaufgabe dar, was wiederum ein Kernanliegen der Disability Studies berührt, die es als ihre Aufgabe ansehen, verbreitete Bilder von Behinderung zu kritisieren, alternative Perspektiven einzubringen und somit Behinderungen abzubauen (Wegner, 2020). Diese Aufgabe richtet sich nicht nur auf den wissenschaftlichen Diskurs, sondern stellt eine an die Öffentlichkeit gerichtete Kommunikationsaufgabe dar, die die Disability Studies wahrzunehmen haben. Um diese Kernaufgabe deutlicher herausstellen zu können, wird hier vorgeschlagen, die Disability Studies um diese Dimension zu ergänzen und dies unter dem Begriff der Public Disability Studies zu verfolgen. Der Begriff der Public Disability Studies dockt begrifflich an andere Wissenschaften an, die diese Aufgabe bereits verfolgen, wie z.B. Geschichtswissenschaften, Medizin oder Theologie und Religionspädagogik (Egger et al., 2020; Grümme, 2018; Hinz \& Körber, 2020; Pirner et al., 2020). Public Disability Studies richten ihren Blick auf die Öffentlichkeit und die Frage, wie dort die Anliegen des eigenen Fachgebiets kommuniziert werden können, sodass gesellschaftlich, medial und damit auch politisch Einfluss genommen werden kann. 
Mit Öffentlichkeit wird ein recht offener Begriff gewählt, der sowohl Medien, als auch unterschiedlichste gesellschaftliche Akteure, den Bildungsbereich sowie Gesetzgeber umfasst. Der Aufgabe, öffentlich über Benachteiligungen und Barrieren zu informieren sowie die zu erforderlichen Prozesse mitzugestalten, könnten sich die Disability Studies, verstanden als Public Disability Studies, annehmen. Dazu gilt es, Öffentlichkeitsarbeit noch stärker zu betreiben, um Themen rund um gleichberechtigte Partizipation und Inklusion öffentlich zu kommunizieren - und dadurch zur kritisch-konstruktiven Nutzung, die als Schlüssel einer barriereärmeren Partizipation identifiziert worden war, beizutragen.

Die Public Disability Studies beschreiben keine neue Subkategorie der Disability Studies, sondern betonen noch einmal stärker die Aufgabe der Öffentlichen Kommunikation - gerade auch vor den Herausforderungen im digitalen Raum. Disability Studies als Public Disability Studies nutzen den digitalen Raum, um über eigene Anliegen zu informieren, um über Barrieren aufzuklären und um im Digitalen Peer-Netzwerke, in denen Menschen sich sowohl in geschützten Räumen vernetzen und offen austauschen können als auch gemeinsam öffentlich für ihre Anliegen auftreten können, zu schaffen. Das heißt Public Disability Studies unterstützen den Aufbau von Plattformen und Kommunikationswegen, die es behinderten Menschen ermöglichen, sichtbar und politisch aktiv zu sein. Barrieren können abgebaut werden, weil Menschen sichtbar und ansprechbar sind, weil heteronormative Bilder hinterfragt und durch Soziale Medien Vergemeinschaftung möglich wird.

Das ist nicht nur handlungsorientiert zu denken, sondern Public Disability Studies richten ihr Interesse auch auf die Erforschung öffentlicher Kommunikationen, wie z.B. die Erforschung ausschließender und grenzverletzender Kommunikation im öffentlichen Raum, wie es z.B. Schwarz-Friesel in Bezug auf Antisemitismus erforscht hat (Schwarz-Friesel, 2018), sowie eine Initiierung eines öffentlichen Diskurses darüber. Dies wäre breiter durchzuführen unter der Frage, wie sich Machtverhältnisse und Diskriminierungen in digitalen Kommunikationen darstellen.

Im Abwägen zwischen dem utopischen und dystopischen Gehalt, der Digitalisierungsprozessen zukommt, wurde deutlich, dass es den Public Disability Studies auch darum gehen muss, die damit verbundenen ethischen Fragen zu thematisieren. Dies in einer allgemeinverständlichen Form zu tun, um die eigenen Anliegen in ganz unterschiedlichen Bereichen einbringen zu können, ist eine wichtige Aufgabe. Welche Auswirkungen Digitalisierung zeitigt, ist dabei breit wahrzunehmen und in der digitalen sowie der analogen Sphäre zu thematisieren, um Ausschlüsse sowie Diskriminierungen aufdecken und beenden zu können.

\section{Fazit}

Der vorliegende Beitrag erörterte das utopische bzw. dystopische Potenzial von mit der Digitalisierung einhergehenden Transformationsprozessen hinsichtlich einer ent- oder behindernden Gesellschaft. Dabei konnte gezeigt werden, dass die Digitalisierung hinsichtlich des Ziels einer nichtbehinderten Partizipation sich sowohl unterstützend als auch hemmend auswirkt - also gleichermaßen Utopie wie Dystopie auszumachen sind.

Als problematisch ist es einzuschätzen, wenn stärker die utopischen Aspekte im Vordergrund stehen, weil die Digitalisierung bessere Zugänglichkeiten sowie assistierende Technologien bietet, und die dystopischen Aspekte dabei weniger sichtbar sind, da die eigentlichen Barrieren in der fehlenden Beteiligung liegen, wenn Menschen außen vor bleiben, da sie nicht angesprochen werden und sich nicht als selbstwirksam erleben können, und die potenziell zur Verfügung stehenden enthindernden Möglichkeiten nicht genutzt werden. Das ist besonders hinderlich, da diese Form des Behindertwerdens nicht auf den digitalen Raum begrenzt ist, sondern mit dem analogen Raum verwoben ist.

,Barrierefreiheit' ist daher komplex anzugehen und umfasst mehr als ein ,Umprogrammieren' von Benutzungsoberflächen. Als Schlüssel für den Abbau von Barrieren wurde das Erschließen von Relevanz, Selbstwirksamkeitserfahrungen sowie das Herstellen einer besseren Passförmigkeit zwischen 
Nutzungsverhalten und digitalen Angeboten identifiziert. Um dieser komplexen Aufgabe gerecht zu werden, die Öffentlichkeits- und Aufklärungsarbeit sowie politische Einflussnahme umfasst, wurde vorgeschlagen, dass die Disability Studies sich hier stärker im Sinne von Public Disability Studies einbringen.

\section{Literatur}

Allport, G. (1971). Die Natur des Vorurteils. Kiepenheuer \& Witsch.

Apple. (2021). Bedienungshilfen iPhone. Apple. https://www.apple.com/de/accessibility/iphone/

Bosse, I., Zaynel, N., \& Lampe, C. (2019). Mediennutzung und Vermittlung von Medienkompetenz in der Behindertenhilfe in Bremen. Ergebnisse der MeKoBe-Studie. Merz. Zeitschrift für Medienpädagogik, 19(5), 24-31. https://www.jff.de/fileadmin/user_upload/merz/cover/2019/merz_519_0925_df_Bosse-etal.pdf

Brehme, D., Fuchs, P., Köbsell, S., \& Wesselmann, C. (2020). Einleitung: Zwischen Emanzipation und Vereinnahmung. Disability Studies im deutschsprachigen Raum. In D. Brehme, P. Fuchs, S. Köbsell, \& C. Wesselmann (Hrsg.), Zwischen Emanzipation und Vereinnahmung. Disability Studies im deutschsprachigen Raum (S. 9-21). Beltz Juventa.

Bundesministerium für Gesundheit. (2013). Abschlussbericht zur Studie Unterstützung Pflegebedürftiger durch technische Assistenzsysteme. https://iegus.eu/publikationen/unterstuetzungpflegebeduerftiger-durch-technische-assistenzsysteme/

Charbonnier, R. (2018). Digitalisierung: Theologische Selbstklärungen und Gegenwartsinterpretationen. Eine Skizze. Zeitschrift für Pädagogik und Theologie, 3, 238-250. https://doi.org/10.1515/zpt-20180030

Cirkel, M. (2020). Digitale Inklusion Älterer. Ein quartiersbezogener Ansatz. Forschung Aktuell, 04, 1-14. https://www.iat.eu/media/forschung_aktuell_2020-04_1.pdf

Coenen, C., Gammel, S., Heil, R., \& Woyke, A. (2010). Einleitung der Herausgeber. In C. Coenen, S. Gammel, R. Heil, \& A. Woyke (Hrsg.), Die Debatte über "Human Enhancement" (S. 9-18). transcript.

Deutschlandfunk Nova. (2018). Gleichberechtigung. Wikipedia soll weiblicher werden. Deutschlandfunk. https://www.deutschlandfunknova.de/beitrag/gender-bias-auf-wikipedia

DGfE Sektion Sonderpädagogik. (2020). Teilhabe auf Distanz? Deutsche Gesellschaft für Erziehungswissenschaft.https://www.dgfe.de/fileadmin/OrdnerRedakteure/Sektionen/Sek06_SondPa ed/2020_Stellungnahme_Corona.pdf

Domsgen, M. (2019). Religionspädagogik. Evangelische Verlagsanstalt.

Düggeli, A., Boéchat-Heer, S., \& Biedermann, H. (2018). Editorial. Digitale Transformation und „Digital Literacy Education“. Neue Forschungsbefunde zu einem herausfordernden Spannungsverhältnis. Schweizerische Zeitschrift für Bildungswissenschaften, 40(2), 299-302.

Egger, M., Razum, O., \& Rieder, A. (Hrsg.). (2018). Public Health Kompakt. De Gruyter. 
Europäisches Parlament, Rat der Europäischen Union. (2016). Richtlinie 2016/2102. Über den barrierefreien Zugang zu den Websites und mobilen Anwendungen öffentlicher Stellen. https://eurlex.europa.eu/legal-content/DE/TXT/PDF/?uri=CELEX:32016L2102\&from=DE

Fabricius, S. (2021). Transhumanismus. In WiReLex - Das wissenschaftliche-religionspädagogische Lexikon im Internet. Abgerufen am 22. März 2021 von https://www.bibelwissenschaft.de/stichwort/200883/

Festl, R. (2017). Mobbing im Internet. DJI-Impulse, 3, 22-24.

Freese, B., \& Mayerle, M. (2015). Digitale Teilhabe als Teil einer barrierefreien (E-)Partizipationskultur am Beispiel des PIKSL-Labors. In M. Düber, A. Rohrmann, \& M. Windisch (Hrsg.), Barrierefreie Partizipation. Entwicklungen, Herausforderungen und Lösungsansätze auf dem Weg zu einer neuen Kultur der Beteiligung (S. 381-395). Beltz Juventa.

Gottwald, C. (2019). Behinderung [online]. In socialnet Lexikon. Abgerufen am 22. März 2021, von https://www.socialnet.de/lexikon/Behinderung

Götz, M. (2019). (K)eine Form des Empowerment? Selbstinszenierung von Influencerinnen auf Instagram. Communicatio Socialis, 52(3), 349-355.

Grümme, B. (2017). Heterogenität in der Religionspädagogik. Grundlagen und konkrete Bausteine. Herder.

Grümme, B. (2018). Aufbruch in die Öffentlichkeit? Reflexionen zum „public turn“ in der Religionspädagogik. transcript.

Harari, Y. (2017). Homo deus. Eine Geschichte von Morgen. C.H. Beck.

Hasebrink, H., Lampert, C., \& Thiel, K. (2019). Online-Erfahrungen von 9- bis 17-Jährigen. Ergebnisse der EU Kids Online-Befragung in Deutschland 2019. Leibniz-Institut für Medienforschung, Hans-BredowInstitut.

Hegemann, L. (2019, 21. November). Weiblich, Ehefrau, kreditunwürdig? ZEIT Online. https://www.zeit.de/digital/datenschutz/2019-11/apple-card-kreditvergabe-diskriminierung-frauenalgorithmen-goldman-sachs/komplettansicht

Hinz, F., \& Körber, A. (Hrsg.) (2020). Geschichtskultur - Public History - Angewandte Geschichte. Geschichte in der Gesellschaft: Medien, Praxen, Funktionen. Vandenhoeck \& Ruprecht.

Hirschberg, M. (2021). Barrieren als gesellschaftliche Hindernisse - Sozialwissenschaftliche Überlegungen. In M. Schäfers, \& F. Welti (Hrsg.), Barrierefreiheit - Zugänglichkeit - Universelles Design. Zur Gestaltung teilhabeförderlicher Umwelten (S. 23-35). Verlag Julius Klinkhardt.

Karlstetter, N. (2018). Bilanzierung von Umwelt- und Sozialfolgen für mehr Nachhaltigkeit: Das Beispiel Fairphone. Eine Analyse der Auswirkungen auf Beschäftigungsstrukturen und Unternehmen. HansBöckler-Stiftung.

Köver, C., \& Reuter, M. (2019, 2. Dezember). Diskriminierende Moderationsregeln. TikToks Obergrenze für Behinderungen. Netzpolitik.org. https://netzpolitik.org/2019/tiktoks-obergrenze-fuer-behinderungen/

Kronauer, M. (2015). Exklusion. Die Gefährdung des Sozialen im hoch entwickelten Kapitalismus. Campus.

Kutscher, N., \& Otto, H. (2014). Digitale Ungleichheit - Implikationen für die Betrachtung medialer Jugendkulturen. In K. Hugger (Hrsg.), Digitale Jugendkulturen (S. 283-298). Springer. 
Lachner, T. (2015, 22. Dezember). Digitale Nomaden auf Kreuzfahrt. „All-inclusive ist schon geil“. Spiegel Online. https://www.spiegel.de/reise/fernweh/digitale-nomaden-auf-kreuzfahrt-all-inclusive-ist-geila-1068661.html

Lanier, J. (2014). Wem gehört die Zukunft. Hoffmann und Campe.

Lanier, J. (2015). Wenn Träume erwachsen werden. Hoffmann und Campe.

Leven, I., \& Utzmann, H. (2019). Die Vielfalt der Digital Natives. In M. Albert, K. Hurrelmann, \& G. Quenzel (Hrsg.), Jugend 2019. 18. Shell Jugendstudie. Eine Generation meldet sich zu Wort (S. 247-312). Beltz.

Lühr, T., Ziegler, A., Vogl, E., \& Boes, A. (2020). \#UmbruchErleben. Wie erleben die Menschen die digitale Transformation? Abschlussbericht des qualitativen Projekts am ISF München. Bidt - Bayrisches Forschungsinstitut für Digitiale Transformation. https://www.bidt.digital/wpcontent/uploads/2020/07/bidt-Studie_UmbruchErleben_Online.pdf

Metzler, C., Jansen, A., \& Kurtenacker, A. (2020). Betriebliche Inklusion für Menschen mit Behinderung in Zeiten der Digitalisierung. IW-Report, 7, 1-31.

Meyer, S. (2016). Technische Unterstützung im Alter - was ist möglich, was ist sinnvoll? Expertise zum Siebten Altenbericht der Bundesregierung. Deutsches Zentrum für Altersfragen. https://nbnresolving.org/urn:nbn:de:0168-ssoar-49980-9

Moosmann, T. (2016, 1. November). Digitale Nomadin: Von Herausforderungen und Vorteilen des Vanoffice. Keine Eile. https://www.keine-eile.de/digitale-nomadin-portugal/

Nida-Rümelin, J., \& Weidenfeld, N. (2018). Digitaler Humanismus. Eine Ethik für das Zeitalter der Künstlichen Intelligenz. Piper.

Patzer, N., \& Pinkwart, N. (2019). Inklusiver Unterricht in Informatik und darüber hinaus: Wie kann inklusives eLearning dazu beitragen? In J. Frohn, E. Brodesser, V. Moser, \& D. Pech (Hrsg.), Inklusives Lehren und Lernen. Allgemein- und fachdidaktische Grundlagen (S. 136-145). Verlag Julius Klinkhardt.

Pirner, M., Lähnemann, J., Haussmann, W., \& Schwarz, S. (Hrsg.) (2020). Public Theology Perspectives on Religion and Education. Routledge.

Rebhorn, D. (2019). Digitalismus. Die Utopie einer neuen Gesellschaftsform in Zeiten der Digitalisierung. Springer.

Schäfer, A. (2015). Selbstbestimmte Leistungssteigerung. Die Diskussion um das pharmazeutische NeuroEnhancement. In A. Schäfer, \& C. Thompson (Hrsg.), Leistung (S. 151-180). Schöningh.

Schenk, S., \& Karcher, M. (Hrsg.) (2018). Überschreitungslogiken und die Grenzen des Humanen. (Neuro-) Enhancement-Kybernetik - Transhumanismus. epubli.

Sendler, U. (2018). Das Gespinst der Digitalisierung. Menschheit im Umbruch - auf dem Weg zu einer neuen Weltanschauung. Springer.

Strasser, P. (2020). Transhumanismus. In W. Schaupp, \& J. Platzer (Hrsg.), Der verbesserte Mensch. Biotechnische Möglichkeiten zwischen Freiheit und Verantwortung (S. 15-28). Nomos.

Schwarz-Friesel, M. (2018). Antisemitismus 2.0 und die Netzkultur des Hasses. Judenfeindschaft als kulturelle Konstante und kollektiver Gefühlswert im digitalen Zeitalter (Kurzfassung). Technische Universität Berlin. https://www.linguistik.tu-berlin.de/fileadmin/fg72/Antisemitismus_2-0_kurz.pdf 
Schwalb, H., \& Theunissen, G. (2018). Inklusion, Partizipation und Empowerment in der Behindertenarbeit. Best Practice-Beispiele: Wohnen - Leben - Arbeit - Freizeit. Kohlhammer.

Thiel, T. (2014). Die Schönheit der Chance: Utopien und das Internet. Juridikum: Zeitschrift für Kritik, Recht, Gesellschaft, 15(4), 459-471.

Tillmann, A. (2014). Girls Media - Feminist Media: Identitätsfindung, Selbstermächtigung und Solidarisierung von Mädchen und Frauen in virtuellen Räumen. In K. Hugger (Hrsg.), Digitale Jugendkulturen (S. 195-208). Springer.

Wegner, G. (2020). Erzählungen vom ,behindert Werden'. Literaturwissenschaftliche Perspektiven auf Behinderung. In D. Brehme, P. Fuchs, S. Köbsell, \& C. Wesselmann (Hrsg.), Zwischen Emanzipation und Vereinnahmung. Disability Studies im deutschsprachigen Raum (S. 189-195). Beltz Juventa.

Windisch, M., Rohrmann, A., \& Düber, M. (2015). Barrierefreie Partizipation - eine Quintessenz. In M. Windisch, A. Rohrmann, \& M. Düber (Hrsg.), Barrierefreie Partizipation. Entwicklungen, Herausforderungen und Lösungsansätze auf dem Weg zu einer neuen Kultur der Beteiligung (S. 396414). Beltz Juventa.

Wimmer, M. (2014). Antihumanismus, Transhumanismus, Posthumanismus: Bildung nach ihrem Ende. In S. Kluge, G. Steffens, \& I. Lohmann (Hrsg.), Menschenverbesserung - Transhumanismus (S. 237-265). Lang.

Witten, U. (2016). \#Selfies und \#Inklusion? Überlegungen zum Inklusionspotenzial von Selfies im Religionsunterricht. In T. Gojny, K. Kürzinger, \& S. Schwarz (Hrsg.), Selfie - I like it: Anthropologische und ethische Implikationen digitaler Selbstinszenierung (S. 165-187). Kohlhammer.

Wolfert, S., \& Leven, I. (2019). Freizeitgestaltung und Internetnutzung: Wie Offline und Online ineinander greifen. In M. Albert, K. Hurrelmann, \& G. Quenzel (Hrsg.), Jugend 2019. 18. Shell Jugendstudie. Eine Generation meldet sich zu Wort (S. 213-246). Beltz.

\section{Autor_in}

Dr. Ulrike Witten ist Professorin für Evangelische Religionspädagogik an der Universität Bielefeld. Ihre Forschungsschwerpunkte sind Inklusion und Religionspädagogik, diakonisches Lernen sowie Religionsdidaktik angesichts religiöser und weltanschaulicher Heterogenität.

E-Mail: ulrike.witten@uni-bielefeld.de 